\title{
Adaptations of Synaptic Form in an Aberrant Projection to the Avian Cochlear Nucleus
}

\author{
Thomas N. Parks, Dwan A. Taylor, and Hunter Jackson \\ Department of Anatomy, University of Utah School of Medicine, Salt Lake City, Utah 84132
}

Surgical removal of the otocyst in chick embryos induces axons from the contralateral cochlear nucleus (nucleus magnocellularis, NM) to form, in addition to their normal endings in nucleus laminaris (NL), anomalous and persistent functional contacts in the ipsilateral NM (Jackson and Parks, 1988). We have examined how interaction between the abnormal synaptic partners during development influences the form of the axon terminal and its relation to the target neuron. In the light microscope, aberrant axon terminals labeled in vitro with HRP appear to form boutons quite unlike the large calycine endbulbs made by the normal cochlear nerve (CN) endings in NM. In the electron microscope, however, the anomalous endings appear embedded in the NM cells, something never seen normally in NM or NL. Morphometric analyses were performed on electron micrographs from NM and NL in animals aged embryonic day $(E) 19$ to posthatching day $(P) 2$ from which the right otocyst had been removed on E3 and in normal control animals. Aberrant endings appose $18 \%$ of the circumference of operated NM cells, versus $45 \%$ for $\mathrm{CN}$ axons in the normal NM at this age. The mean length of membrane apposition for the anomalous NM-to-NM endings was $215 \%$ greater than for normal NM-to-NL endings but $54 \%$ smaller than that in normal $\mathrm{CN}$ endings. These results support the idea that developmental interactions between synaptic partners can influence the form of the contact between the 2 neurons. The results also demonstrate, however, that formation of persistent and functional synapses with NM neurons throughout development is not sufficient to induce any axon to assume the calycine form of a cochlear nerve endbulb. Thus, target influences on presynaptic differentiation are clearly limited in their scope.

The various branches of a single axon can form morphologically heterogenous endings on different target neurons (e.g., Lorente de No, 1981) and axons of different origin ending on the same target can have a similar specialized form (e.g., Palay and ChanPalay, 1974). For these reasons, it has been concluded that the postsynaptic cell controls the form of the presynaptic axon (Mugnaini, 1971). Since specializations in the form of axonal endings are likely related to functional specializations in the transfer of information between neurons, the mechanisms by which axon terminals acquire characteristic shapes and sizes are

\footnotetext{
Received Aug. 9, 1989; revised Sept. 21, 1989; accepted Sept. 27, 1989.

We thank Drs. D. O. Frost and C. von Bartheld for helpful comments. This work was supported by grant DC 00144 from the USPHS.

Correspondence should be addressed to Thomas N. Parks at the above address. Copyright (c) 1990 Society for Neuroscience $0270-6474 / 90 / 030975-10 \$ 02.00 / 0$
}

deserving of study. The supposition that the target cell controls the form of its axonal partners could best be evaluated by directing a novel afferent to form synapses with a target whose normal afferent axons have a characteristic form different from that of the anomalous afferents. Despite the many examples of synapse replacement and rearrangement that have been studied during the last 20 ycars (Cotman, 1985), there are very few cases where axons have been directed in substantial numbers to form persistent synapses with abnormal target neurons. The best studied examples are the aberrant thalamic synapses formed by retinal axons after neonatal ablation of the superior colliculus (Schneider, 1981; Campbell and Frost, 1988) and the anomalous auditory connections formed between the cochlear nuclei $(\mathrm{CN})$ on the 2 sides of the brain after early extirpation of the otocyst in chick embryos (Jackson and Parks, 1988).

In the nucleus magnocellularis (NM) of birds, the cochlear nerve forms very large calyx-like endbulbs on the somatic surface of adendritic or parvodendritic neurons. In contrast, the axons of NM cells normally end in small sprays of boutons on the dendrites of neurons in nucleus laminaris (NL) (Rubel and Parks, 1988). After early destruction of the $\mathrm{CN}$ rudiment, an anomalous, functional and persistent projection is formed in the deafferented NM by new branches of contralateral NM axons (Jackson and Parks, 1988). The present study has compared the morphologies of cochlear nerve, NM-to-NL, and anomalous NM-to-NM endings to determine the extent to which the interaction of novel synaptic partners influences the form of the resulting contact. A preliminary report of the main findings has been given (Parks et al., 1986).

\section{Materials and Methods}

Surgery. The right otocyst was removed surgically from white Leghorn (Babcock strain) chick embryos on embryonic day (E) 3 as described in a previous study (Parks, 1981a). Operated and normal control animals were allowed to survive until E14 through posthatching day (P) 2 and were then divided into 2 groups for further study. One group of operated animals (E19-P2) and all of the controls were deeply anesthetized with pentobarbital and then perfused transcardially with a trialdehyde fixative as described in a previous study (Parks et al., 1983). After a careful inspection of the skull and brain to verify the complete absence of the inner ear and 8th cranial nerve on the right side, tissue blocks containing NM or NL were processed for conventional transmission electron microscopy. To ensure adequate sampling throughout NM and NL, samples were taken from approximately the 20th, 50th, and 90 th posterior-to-anterior percentile positions within the nuclei (50th and 90th percentiles in normal controls) using the following procedures. Fixed brain stems were sectioned serially in the coronal plane at $100 \mu \mathrm{M}$ on a vibratome. The sections were stained lightly in phosphate buffer containing methylene blue dye. Under a dissecting microscope, small pieces of tissue containing NM or NL at the 3 sampling levels on the left and right sides of the brain were dissected and then processed for routine 
electron microscopy as described previously (Parks, 1981 b; Parks et al., 1983).

In vitro HRP labeling. A second group of operated animals was studied by means of an in vitro brain-stem preparation and labeling of NM axons with HRP (Jackson and Parks, 1982, 1988). In brief, brain stems of anesthetized animals were dissected free of the skull in cold oxygenated avian Tyrode solution, the cerebellum was removed, and the remaining piece of the brain stem glued to the bottom of a perfusion chamber. While the brain stem was perfused with oxygenated avian Tyrode solution at room temperature, HRP was introduced into the crossed dorsal cochlear tract, which carries axons from each NM to the contralateral side of the brain. In animals aged E19-P2, HRP staining of NM axons was achieved by placing a small crystal made of HRP (Sigma Type VI) and Triton X-100 detergent (Escher et al., 1983) into a small cut made in the crossed dorsal cochlear tract. For animals younger than E19, HRP was introduced by iontophoretic injection as previously described (Jackson and Parks, 1988).

Histology. Two hours after application of HRP, the brain stem was fixed for $2 \mathrm{hr}$ in $4 \%$ glutaraldehyde (for light microscopy) or the trialdehyde fixative (for electron microscopy). Transverse sections of this tissue were cut at $100 \mu \mathrm{M}$ and reacted for HRP by the cobalt-intensified diaminobenzidine method of Adams (1981). As described previously, HRP-stained tissue was then processed for light microscopy (Jackson and Parks, 1988) or conventional transmission electron microscopy (Parks, 1981b; Parks et al., 1983).

Morphometric analyses of electron micrographs. Quantitative comparisons were made among a number of groups of E19-P2 animals. To minimize the effects of interanimal variation and to obtain the best quality tissue for quantitative analyses, the main body of observations was made on tissue obtained from the experimental animals fixed by cardiac perfusion. This choice raised 2 potential problems: (1) how to insure that NM neurons on the unoperated left side of the brain in experimental animals are not different from normal NM cells in ways that would preclude their use as valid controls, and (2) how to identify the NM-to-NM endings in unlabeled tissue. In operated animals, the right cochlear nerve is absent and the left nerve is present. Although previous work has shown unequivocally that the number and crosssectional area of neurons in one NM are not affected by removal of the contralateral otocyst (Parks, 1979) and that the morphology of NL dendrites connected to the left NM is similarly unaffected (Parks, 1981a), it was not known if ultrastructural features of NM neurons and their endings in NL are also normal. Morphometric data from normal control animals were thus compared with the left-side data from experimental animals to validate the latter.

Although it is not difficult to distinguish anomalous NM-to-NM endings from the principal noncochlear (NC) endings in NM, which have synaptic densities and synaptic vesicles characteristic of inhibitory endings (Parks, 1981b), some confusion with a minor NC ending type in unlabeled tissue is conceivable. In 30- to 60-d-old hatchling chickens, about $5 \%$ of the circumference of NM neurons is apposed by small axonal endings that can be distinguished from $\mathrm{CN}$ endings only by selective staining or elimination of the $\mathrm{CN}$ fibers (Parks, $1981 \mathrm{~b}$; Code and Rubel, 1989). To determine if our sample of NM-to-NM endings was significantly contaminated by inclusion of endings with different morphological features, we compared morphometric values for NM-toNM endings in unstained perfusion-fixed tissue with those obtained for a sample of HRP-labeled NM-to-NM endings from in vitro preparations.

For quantitative analyses, electron micrographs were used in ways similar to those in previous studies of NM and NL (Parks, 1981 b; Parks et al., 1983). Montages of electron micrographs (at $\times 16,200$ final magnification) were used to calculate the proportion of the ccll body circumference occupied by endings of different types and the length of membrane apposition between each axonal ending and the target cell body. The only criteria used to select neurons for photography were that they be sectioned through the nucleus, well-stained, and free of any large debris or damaged areas that might obscure synaptic endings. Axosomatic endings on NM neurons in normal animals and on the left side of operated animals which had clearly distinguishable synaptic densities and synaptic vesicles associated with them were classifed as $\mathrm{CN}$ endings or NC endings according to criteria described in a previous paper (Parks, $1981 \mathrm{~b}$ ). In the operated right NM, endings were similarly classified into NM-to-NM and NC types. NM-to-NL endings were identified in NL according to previously published criteria (Parks et al., 1983). Since all of these endings have characteristic morphologies, there were very few cases where identification was difficult.
Apposition length values were measured for the region where the preand postsynaptic membranes were in closest apposition. All somatic membrane apposed by the embedded NM-to-NM endings (see below) was included in cell circumference measurements. All measurements were made with an electronic planimeter.

In 4 operated animals aged E19-P2, 64 montages of NM cells were studied; they yielded morphometric data on $139 \mathrm{CN}$ endings, $242 \mathrm{NC}$ endings and 111 NM-to-NM endings. Thirty-six NM-to-NL endings were also studied at $\times 16,200$ in 5 operated animals. Sixty-eight HRP labeled NM-to-NM endings from 8 operated animals were studied. In 3 normal perfusion-fixed control animals, 31 cell montages were studied. These yielded measurements of $151 \mathrm{CN}$ endings, $67 \mathrm{NC}$ endings, and 93 NM-to-NL endings. In all, data were obtained from 14 operated animals and 3 normal controls. The principal comparisons were made among CN, NM-to-NL, and NM-to-NM endings (Figs. 1, 2).

Statistical procedures. For statistical analyses, data were averaged for each montage or sampling site and then combined into means for each animal; these animal means were used for calculating grand means (and standard errors). Nonparametric procedures (2-tailed) were used for inferential statistical comparisons. For within-animal comparisons, the Wilcoxon matched-pairs signed-ranks test was used and for betweenanimal comparisons the Mann-Whitney test. All statistical analyses were performed using StatView II statistical software (Abacus Concepts, Inc.)

\section{Results}

\section{Qualitative observations}

Comparison of low-power electron micrographs from NM on the left and right sides of the brain revealed obvious differences in the proportion of the cell body surface apposed by axonal endings and in the size and location of these endings (Figs. 3, 4). As shown in Figures $1 A, 2$, and 3, most of the normal NM soma is apposed by large axonal endings from the $\mathrm{CN}$ and a few smaller NC endings. The $\mathrm{CN}$ endings are indented by the short somatic processes which stud the cell surface (Jhaveri and Morest, 1982a). In the right NM (Fig. 4), the somatic processes are greatly reduced in number, large areas of the cell body are unapposed by any axonal ending and contain no membrane specializations such as the postsynaptic densities associated with synapses, and several medium-sized endings are seen embedded deeply within the cell body (Figs. 1C, 4; see also figure 6 in Jackson and Parks, 1988). This pattern is found routinely in the right NM but never on the left side or in normal animals. The axosomatic endings in the right NM that are labeled by in vitro HRP injection resemble the embedded endings seen in perfusion-fixed animals (Fig. 5).

\section{Neuronal circumference}

Quantitative analyses of electron micrographs substantiated and extended the qualitative observations. NM neurons on the right side of operated animals were smaller than those receiving normal CN input. Mean (SEM) circumference values for normal control, left-side operated, and right-side operated groups were $81.9(2.8), 88.7(3.3)$, and $77.2(1.8) \mu \mathrm{m}$, respectively; the $13 \%$ left-right difference in operated animals was marginally significant $(z=1.83, p<0.07)$. Since the normal and left-side operated values did not differ, they were combined for an overall "normal" mean of $85.8(2.5) \mu \mathrm{m}$. For the purposes of the following calculations, we make the assumption (which is very nearly true) that NM cells are spheres and calculate the radius $(r)$ of a cell from its circumference $(c)(r=c / 2 \pi)$. The surface area of the cell is then given by $4 \pi r^{2}$. Normal NM neurons and right-side operated neurons in this study had mean surface areas of 2324 and $1901 \mu \mathrm{m}^{2}$, respectively, for a mean difference of $18 \%$. This difference is comparable with the previously reported 

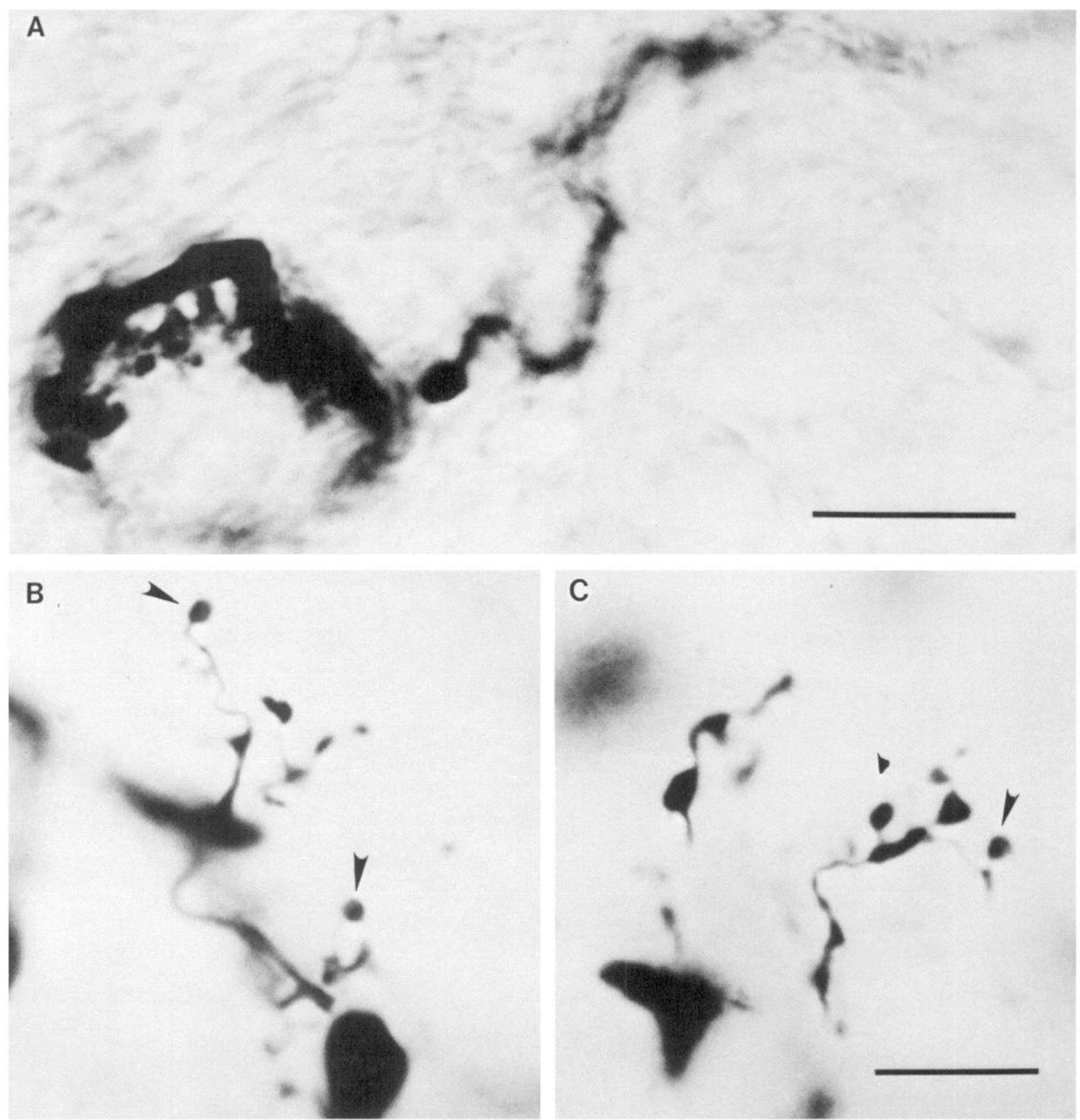

Figure 1. Light micrographs of typical HRP-stained axonal endings. $A$, Cochlear nerve axon forms a calycine ending on a NM neuron in a normal posthatching day (P) 2 animal. Scale bar, $10 \mu \mathrm{m}$. B, Spray of small boutons from a normal NM axon ending in NL. $C$, Boutons in the right NM formed by an axon from the left NM. Scale bar (for $B$ and $C$ ), $10 \mu \mathrm{m}$.

$15 \%$ decrease in neuronal cross-sectional area in NM on the right side of operated animals at this age (Parks, 1979).

\section{Proportion of the neuronal surface occupied by each type of ending}

Analysis of the proportion of neuronal circumference occupied by axonal endings revealed that normal control animals and the left side of operated animals had, respectively, $41.5(1.4) \%$ and $47.2(4.8) \%$ of their circumferences occupied by $\mathrm{CN}$ endings; these proportions did not differ reliably, and the overall mean for these 2 "normal" groups is $44.8(2.9) \%$. The proportions of normal and left-side operated NM neurons occupied by NC endings also did not differ significantly; the mean (SEM) value for these 2 "normal" groups combined is $6.0(1.5) \%$. Thus, on average, normally innervated NM neurons had $50.8 \%$ of their circumference occupied by $\mathrm{CN}$ and $\mathrm{NC}$ endings. In contrast, NM neurons on the operated side of experimental animals had only $25.5 \%$ of their circumference occupied by axonal endings; $18.4(1.7) \%$ was occupied by NM-to-NM endings and $7.1(3.1) \%$ by $\mathrm{NC}$ endings. These findings are illustrated in Figure 6.

\section{Distribution of the NC endings}

It was of interest to evaluate whether NC endings had enlarged their coverage of NM cells in the absence of $\mathrm{CN}$ endings. Since the circumference of NM cells on the operated side is $13 \%$ below control values, it is possible to consider NC coverage in relation 

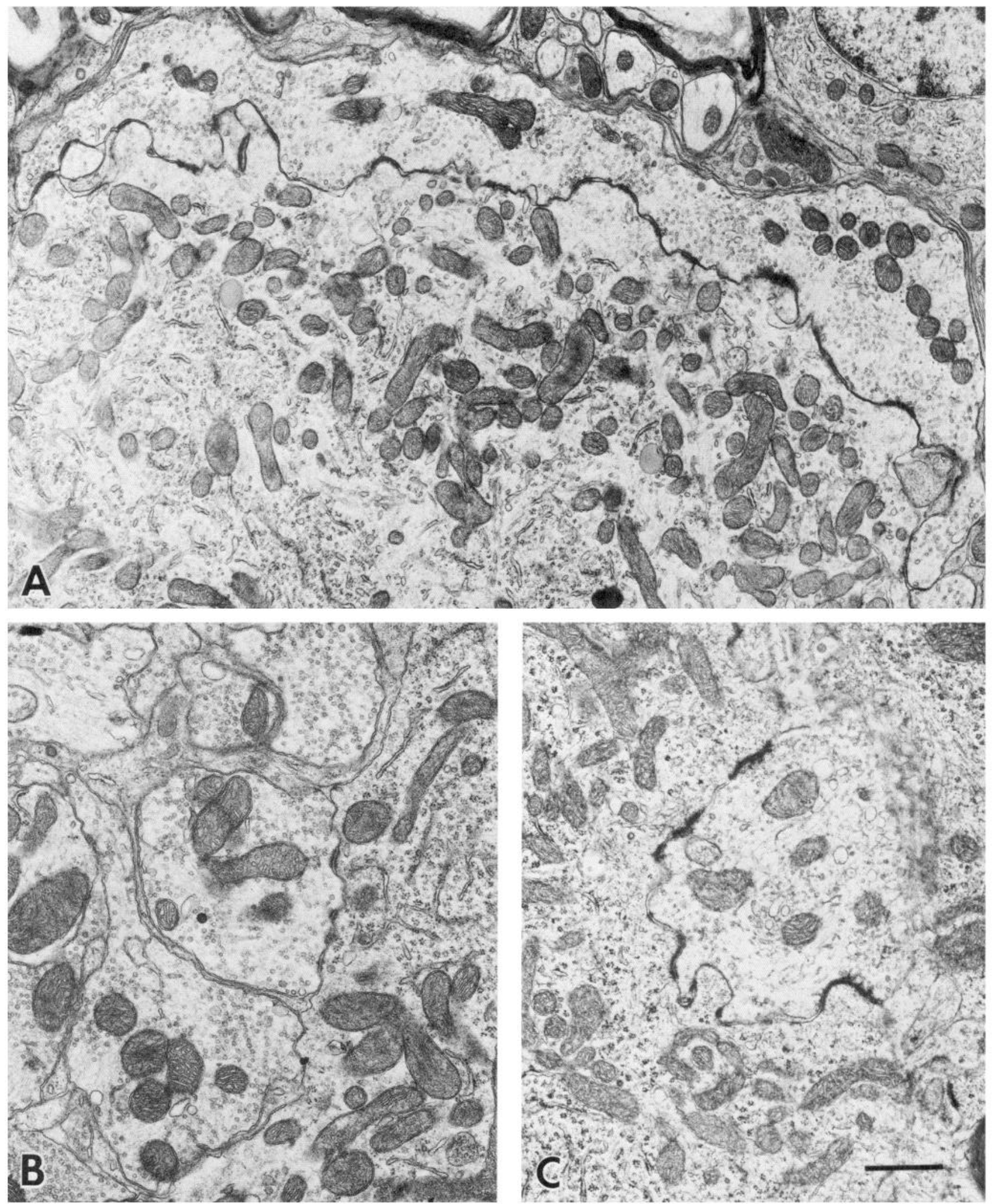

Figure 2. Electron micrographs, taken from a P1 experimental animal, showing qualitatively representative examples of the 3 axonal ending types considered in detail in this study. $A$, Large calycine ending formed by a CN fiber on a nucleus magnocellularis (NM) neuron on the unoperated left side of the brain. Note the short somatic stubs which indent the afferent ending. $B$, Typical boutonal axosomatic ending formed by a NM fiber on its normal target in the right ventral nucleus laminaris. $C$, An induced NM ending deeply indenting a NM cell body. Scale bar, $1 \mu \mathrm{m}$. 


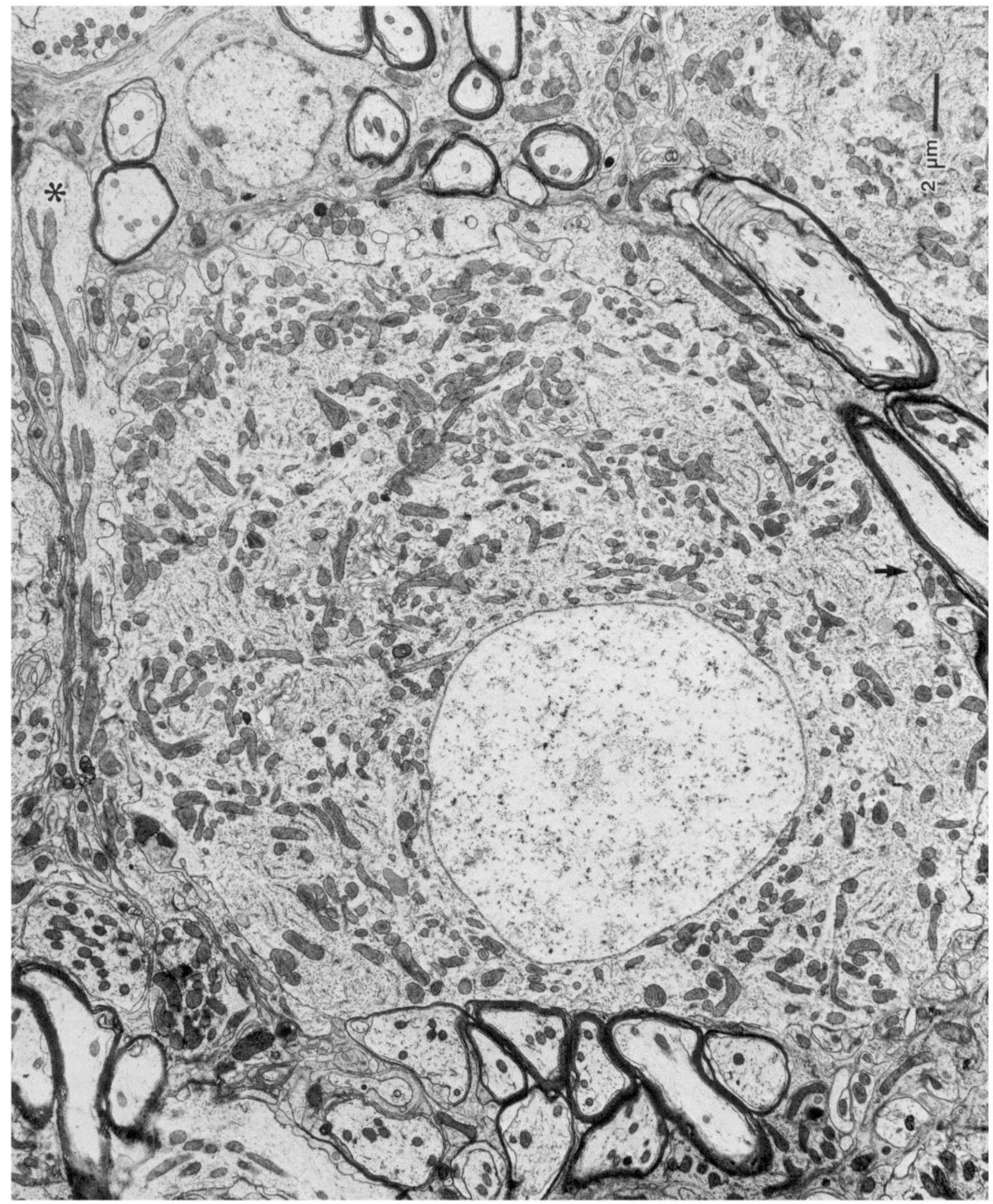

Figure 3. Low-power electron micrograph of a normally innervated NM neuron from an E19 chick embryo to show the normal synaptology. From the upper left (asterisk), a large-caliber $\mathrm{CN}$ axon can be seen forming a characteristic endbulb on the cell body; another endbulb covers the top of the cell. Note the numerous somatic stubs which indent the endbulbs and the extensive coverage of the cell circumference by axosomatic endings. An arrow at the right of the figure indicates the position of a characteristic small NC ending. 

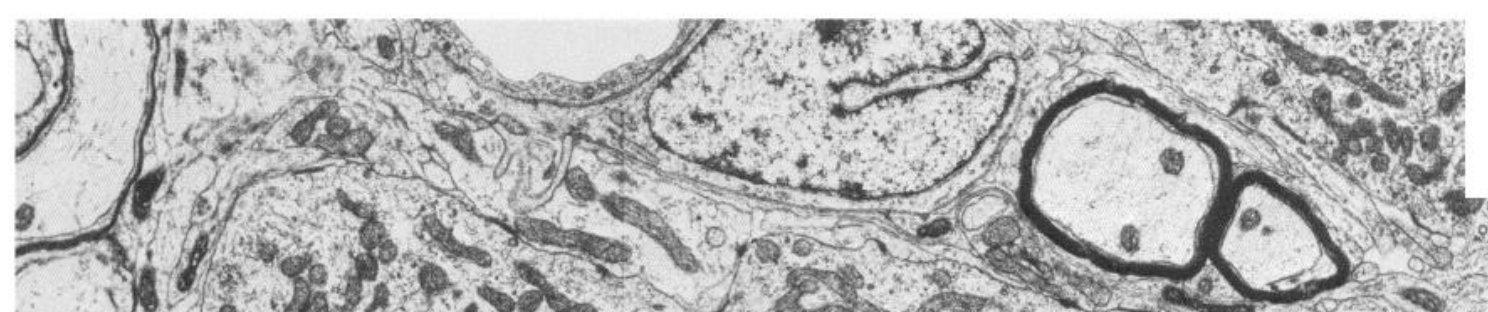

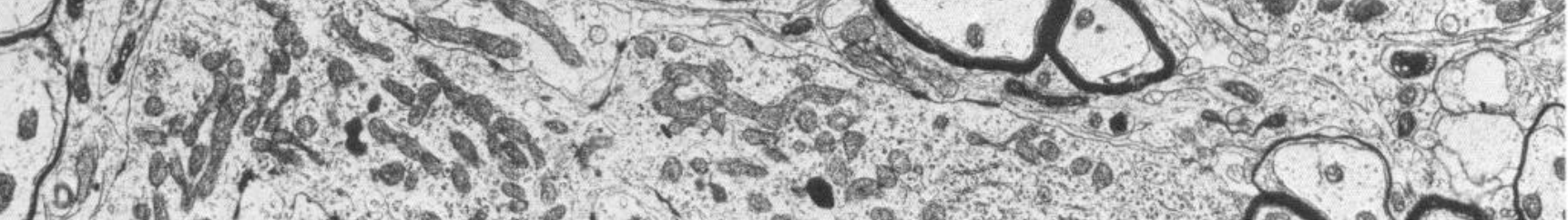

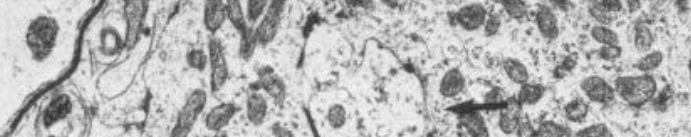

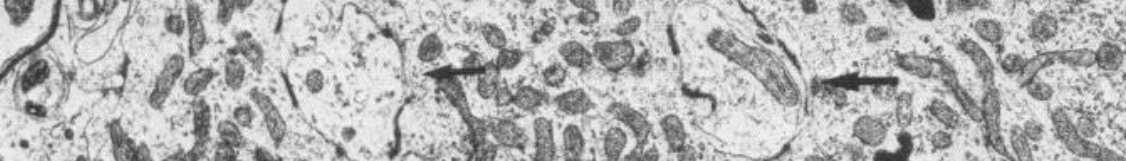

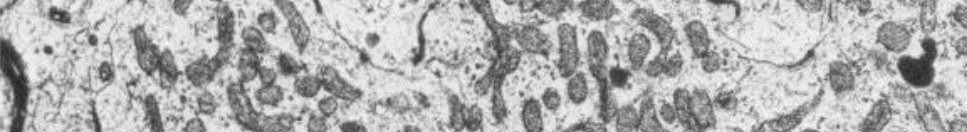

.

Pi

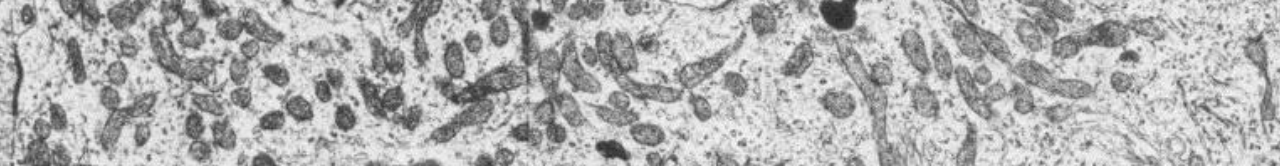

PI.

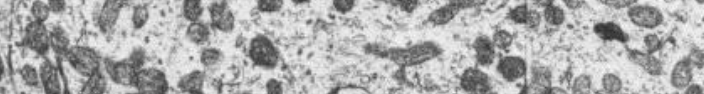

10

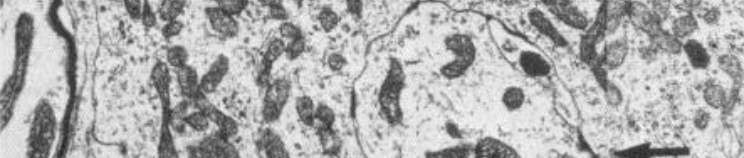

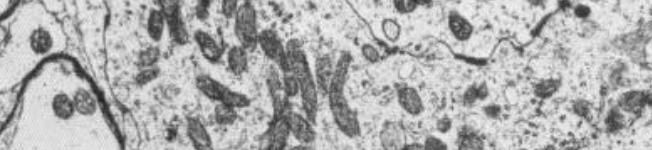

(

nit?

(1)

17

$2+2$

(1) 1

Q.

(1)

-. 2,0 ,

(5)

58

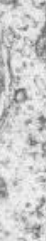

(1)

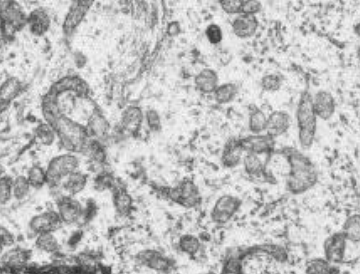

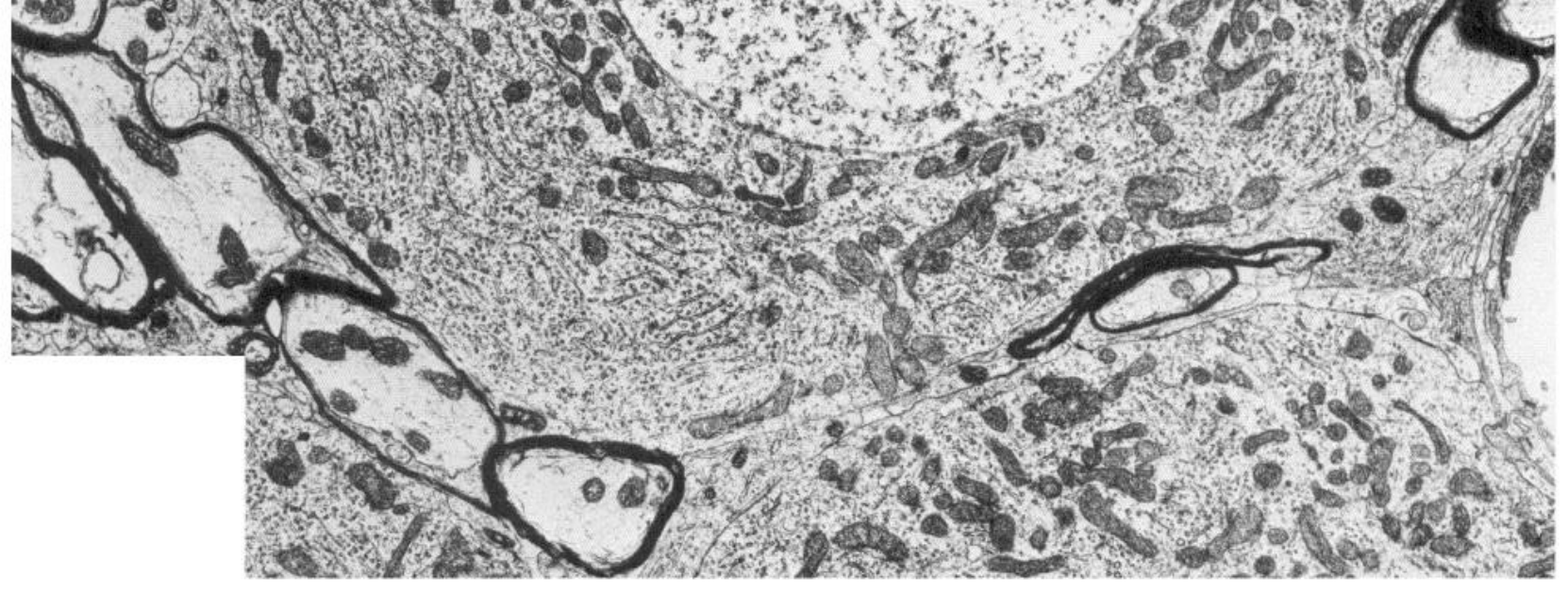




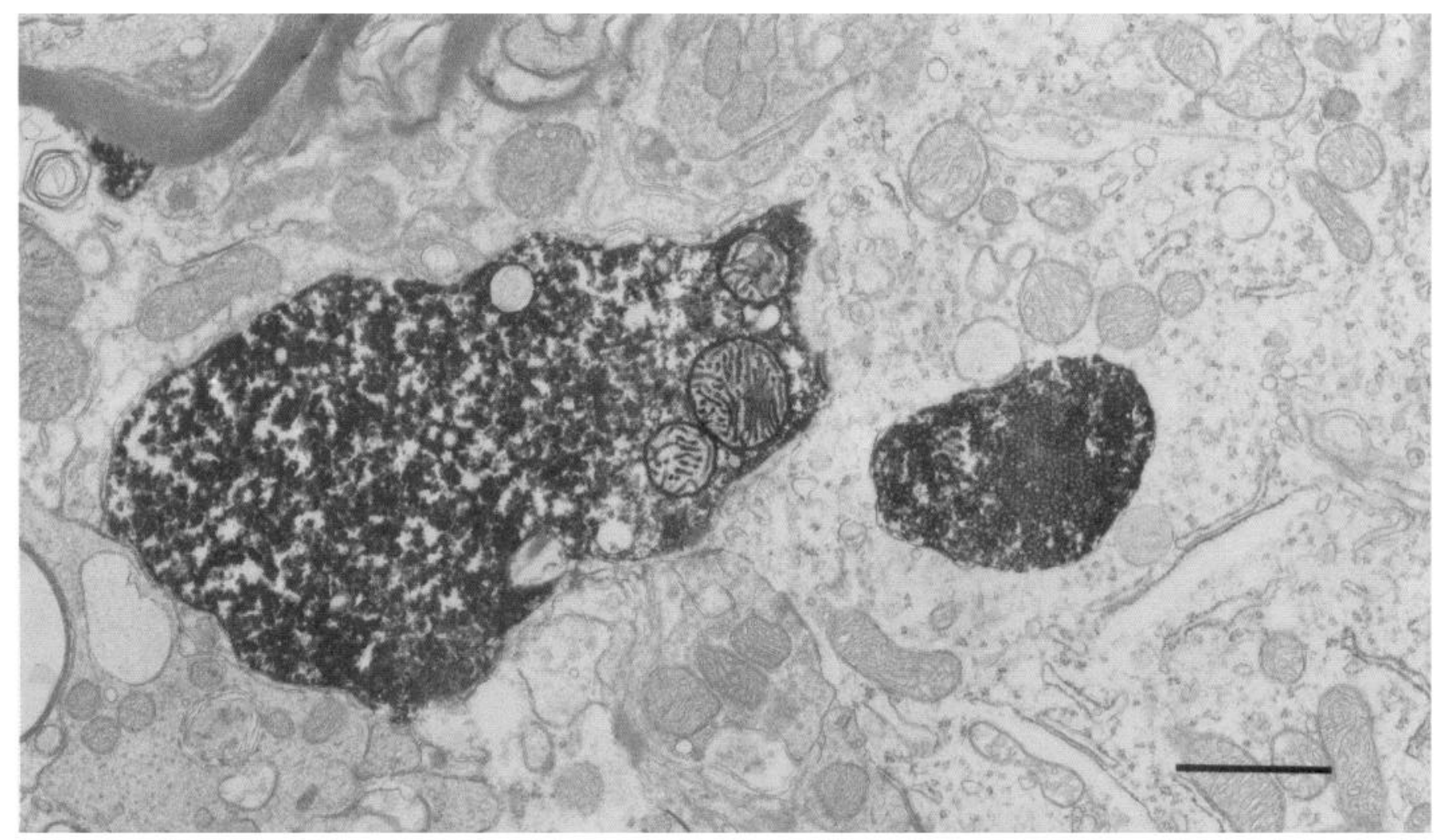

Figure 5. Electron micrograph showing HRP-stained profiles of aberrant NM axon terminals in the contralateral NM; note that the stained profile on the right is embedded within the cytoplasm of its target neuron (cf. Fig. 4).

to $100 \%$ of the operated cell circumference or to $87 \%$ of the normal control circumference. In the first case, $\mathrm{NC}$ coverage is $7.1(3.1) \%$ versus the control value of $6.0(1.5) \%$; this difference is not statistically reliable. If NC coverage is normalized to control cell circumference, the value of $6.2(2.7) \%$ is also not significantly different from controls. Although there was no reliable difference in the proportion of normal and operated NM neurons occupied by $\mathrm{NC}$ endings, the overall $50 \%$ difference between the proportions of the cell circumference occupied by all types of axonal endings in normal and operated neurons was marginally reliable $(z=1.83, p<0.07)$.

\section{Length of membrane apposition}

The length of membrane apposition for $\mathrm{CN}$ endings was 8.9 (1.0) $\mu \mathrm{m}$ in normal control animals and 9.8 (1.2) $\mu \mathrm{m}$ on the left side of operated animals; since these values did not differ reliably, they were combined for an overall "normal" value of 9.4 (0.8) $\mu \mathrm{m}$ (Fig. 5). Similarly, the mean apposition lengths of NC endings in normal control and left-side operated animals did not differ significantly and could be combined into a single value of $1.5(0.1) \mu \mathrm{m}$ (Fig. 5). This normal value did not differ significantly from the NC apposition length on the right side of operated animals, $1.6(0.2) \mu \mathrm{m}$. Endings in NL arising from NM neurons have mean apposition lengths of $2.1(0.2) \mu \mathrm{m}$ in normal control animals and $1.9(0.2) \mu \mathrm{m}$ in left-side operated animals; since these values did not differ reliably, they were combined for a grand mean of $2.0(0.1) \mu \mathrm{m}$. Anomalous NM-to-NM endings were studied in both perfusion-fixed and HRP-labeled operated animals; mean apposition lengths of these 2 groups were identical $(4.3 \mu \mathrm{m})$ and were thus combined for a mean of 4.3 $(0.2) \mu \mathrm{m}$. As illustrated in Figure 7, apposition length in the NM-to-NM endings was significantly different from those of both $\mathrm{CN}$ endings $(z=3.56, p<0.001)$ and NM-to-NL endings $(z=3.70, p<0.001)$.

\section{Discussion}

Comparison with previous studies on the chick auditory nuclei There are 2 instances in which the current measurements differ significantly from previously reported ones. The present data show a mean apposition length of $9.4 \mu \mathrm{m}$ for $\mathrm{CN}$ endings in E19-P2 animals, whereas Parks (1981b) reported a value of 4.8 $\mu \mathrm{m}$ in P30-60 animals. Similarly, the proportion of the normal cell circumference occupied by CN endings was $44.8 \%$ in E19P2 animals and $65.8 \%$ in P30-60 animals. These differences are consistent with the major changes in the shape and size of $\mathrm{CN}$ endbulbs that are known to occur during development (Ryugo and Fekete, 1982). As described by these authors for the cat, endbulbs undergo 3 sequential stages of morphological development between postnatal days 2 and 20 . In the first stage, the endings are solid cuplike structures with few branches. In the

Figure 4. Montage of electron micrographs through a NM neuron on the deafferented right side of the same E19 animal illustrated in Figure 3. In comparison with Figure 3, note the relative absence of axosomatic endings, the smooth contours of the neuronal surface, the absence of somatic membrane specializations except opposite axonal endings and (arrows) the induced NM-NM endings deeply embedded within the cell soma. 


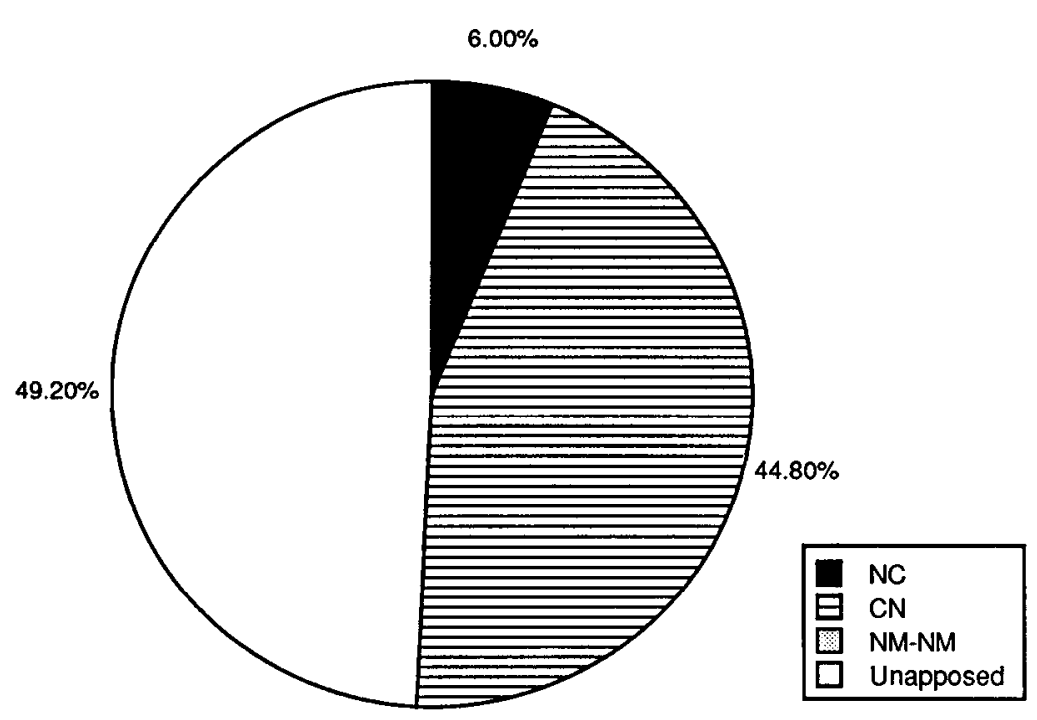

Normal

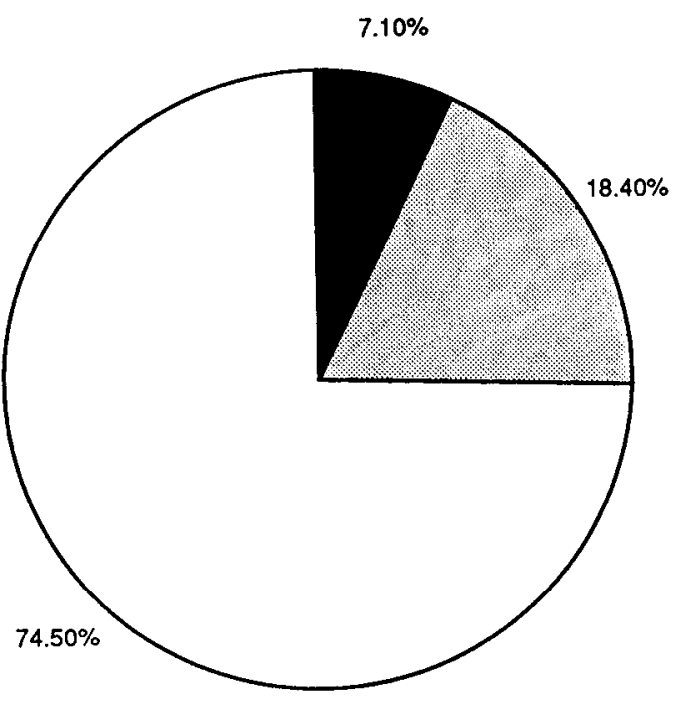

Operated

Figure 6. Mean percentage of the circumference of NM neurons occupied by CN, NC, and NM-NM axosomatic endings on the right side of the brain in operated animals and in normal controls. The proportion of the cell circumference unapposed by any axonal endings is also shown.

second stage, the endings retain some broad solid regions but also become fenestrated and branched. In the third stage, which persists into adulthood, $\mathrm{CN}$ endings form a reticulum of small branches enveloping their target neurons. Since this pattern of development is supported by our unpublished data for chickens, it would be expected that mean apposition length of $\mathrm{CN}$ endings would be greater in embryos than in older animals and that the proportion of the cell body occupied by $\mathrm{CN}$ endings would increase over the coursc of devclopment.

Young and Rubel (1986) report that one fully reconstructed normal NM axonal arbor (from a sample of 119 reconstructions) produced, in addition to its other projections, a single small bouton on another NM cell (their figure 13). Thus, the possibility must be considered that some of the NM-to-NM endings we studied arise from the ipsilateral rather than the contralateral NM. In this regard, several points deserve discussion. First, despite the existence of hundreds of HRP-labeled NM neurons (from 2 laboratories) in which such a projection should be evident (Jackson and Parks, 1982, 1988; Young and Rubel, 1983, 1986; Parks and Jackson, 1984), ipsilateral NM-to-NM axosomatic contacts are seen only very rarely in operated or normal animals. Second, since in normal animals at least $95 \%$ of the surface of NM cells has been shown to be occupied either by axosomatic endings of known origin or by no endings at all (Parks, 1981b; Code and Rubel, 1989; Code et al., 1989), there is little reason to believe that a quantitatively significant ipsior contralateral axosomatic NM-to-NM projection, either of which should be glutaminergic (Jackson and Parks, 1988), exists normally.

Third, it is clear from the anatomical and electrophysiological results of Jackson and Parks (1988) and the present HRP-labeling experiments that a large number of anomalous endings in the right NM do arise directly from neurons in the left NM. Fourth, there is no evidence in our data for more than one mode in the distribution of any morphometric variable for the class we term "NM-to-NM" endings. Thus, there is no objective reason to believe that this class, which differs significantly from
$\mathrm{CN}$ and NM-to-NL endings in the morphometric measures, contains subclasses of ending which differ on these measures. Therefore, even in the very unlikely event that a significant proportion of the NM-to-NM endings measured in this study were ipsilateral ones induced by otocyst removal, the main conclusions of the study would still be valid.

\section{Control of axon terminal form by target neurons}

The present results show that foreign axons induced to form functional synapses with NM neurons do not acquire the gross form of a $\mathrm{CN}$ calyx ending. This is particularly surprising given that the NM-to-NM axons are present in the target nucleus by at least E7 (several days prior to the time of normal synapse formation between the $\mathrm{CN}$ and $\mathrm{NM}$ neurons) and thus can presumably undergo a full range of developmental interactions with their target neurons (Parks et al., 1987). This finding has important implications for understanding how different branches of a single axon (such as the $\mathrm{CN}$ ) can form a variety of morphologically distinct ending types on their various target neurons.

There are a number of well-studied cases in normal animals where single axons make morphologically quite distinct endings on various target cell types. Single climbing fibers of the cerebellum, for example, can make small globose varicositics on granule cell dendrites, large efflorescent endings in the glomeruli and on Golgi neurons, and smooth or beaded tendrils on the cell bodies and proximal dendrites of Purkinje cells (Palay and Chan-Palay, 1974). Similar variations in ending morphology are found in various normal targets of retinofugal axons (see Campbell and Frost, 1988) and CN fibers (Lorente de No, 1981). A related example is the synapse en marron of the cerebellar Golgi neuron, where either climbing fibers or mossy fibers form a large terminal efflorescence on the corrugated surface of the Golgi cell (Palay and Chan-Palay, 1974). Since "a single fiber ending on different types of cells forms different types of terminals, while fibers of different origins ending on corresponding 
portions of cells of the same class may form similar endings," it has been generally accepted that "the modeling of the preand postsynaptic structures seems to depend largely on the postsynaptic element" (Mugnaini, 1971, pp. 148-149). This conclusion, which has been based on studies of normal material, receives important support from the recent experimental work of Campbell and Frost (1988), who find that developing retinal axons induced to form synapses in auditory or somatosensory thalamic nuclei adopt many features characteristic of normal auditory and somatosensory (but not visual) afferent axonal endings.

Of the several morphologically distinct ending types made by various branches of each cochlear nerve axon (Lorente de No, 1981), the large calycine endbulbs in the anteroventral cochlear nucleus of mammals and nucleus magnocellularis of birds and reptiles are the most remarkable. They are among the largest and most specialized axonal endings in the nervous system and appear designed to subserve extraordinarily faithful synaptic transmission between ear and brain (e.g., Goldberg and Brownell, 1973; Ryugo and Fekete, 1982; Hackett et al., 1982). In the 1 - to 2-month-old hatchling chicken, each endbulb covers about one-third of the surface of an NM neuron and each neuron receives, on average, 2 such endings (Parks, $1981 \mathrm{~b}$; Jackson and Parks, 1982). Synaptic transmission at the CN-NM synapse is mediated by an excitatory amino acid neurotransmitter acting on kainate-preferring postsynaptic receptors (Nemeth et al., 1985). Stimulation of the afferent axon produces a very rapid and large excitatory postsynaptic potential in the NM neuron (Hackett et al., 1982), reflecting the powerful excitatory action of the $\mathrm{CN}$.

In maturity, the CN-NM junction presents a deceptively uncomplicated picture of an immense axon terminal cupping a spherical and adendritic or parvodendritic nerve cell body (Parks, $1981 \mathrm{~b}$; Jhaveri and Morest, 1982a). These cells follow a tortuous developmental course to this simple result, however. Prior to E15, CN axons are highly branched and contact a number of multipolar NM neurons; each NM neuron receives functional input from a number of $\mathrm{CN}$ axons on its many long dendrites. Between E13 and E16, CN axons are reduced to a single unbranched fiber ending in a calyx, functional convergence onto each NM neuron is concomitantly reduced by $50 \%$, and NM neurons are transformed from multipolar to unipolar form (Jackson and Parks, 1982; Jhaveri and Morest, 1982b). These remarkable parallel transformations suggested that interaction between these particular afferent axons and target cells might be essential to the development of their characteristic forms (Jhaveri and Morest, 1982b). A previous experimental test of this hypothesis showed that surviving NM neurons do not require the presence of $\mathrm{CN}$ axons to achieve an essentially normal form (Parks and Jackson, 1984), even though 30\% of NM neurons die under these circumstances (Parks, 1979).

Campbell and Frost (1988) have suggested that the differentiation of morphological features involved in contacts among neurons is responsive to interactions among the connected elements. The present data support this general statement in that the experimental coupling of anomalous presynaptic and postsynaptic neurons results in a morphologically anomalous functional contact between the 2 cells. Our results clearly show, however, that there are significant limits to target-controlled morphogenesis of axon terminals. That NM axons innervating NM retain the boutonal form characteristic of their terminals in NL shows that interaction with transforming NM neurons

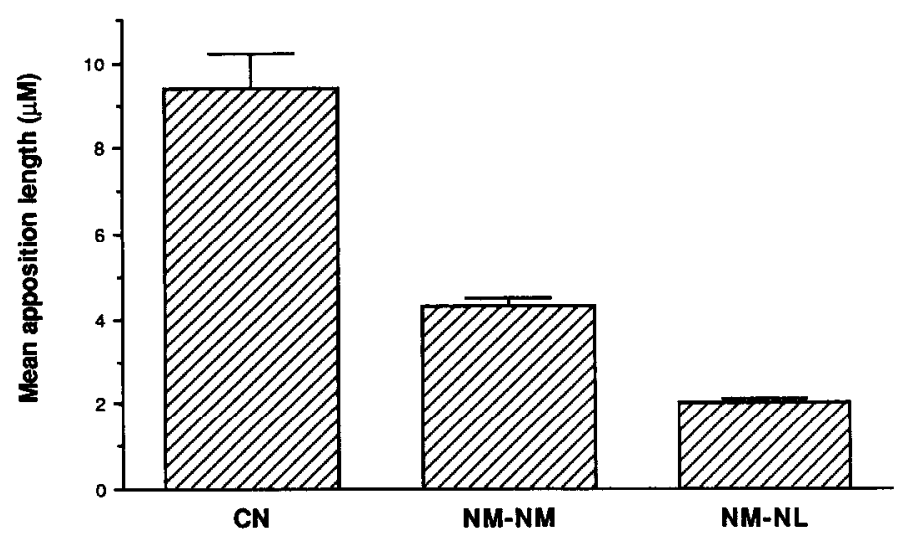

Figure 7. Mean length (and SEM) of the membrane apposition between axonal ending and target neuron for 3 types of axosomatic ending in the avian auditory system. $C N$, normal cochlear nerve endings on nucleus magnocellularis $(N M) ; N M-N M$, the abcrrant projection; $N M-N L$, normal NM endings in nucleus laminaris.

during development is not sufficient to induce any axon to form a calyx-like ending.

It is also true, of course, that the NC endings retain a boutonal form in the absence of CN endings. This finding is less significant than it may first appear, however, since it is known that these NC endings arrive in NM only at about E16 (Jhaveri and Morest, $1982 \mathrm{~b}, \mathrm{c}$ ), after the major morphological transformations of $\mathrm{CN}$ endings and NM neurons are completed. Thus, the finding that NC endings retain a boutonal form after otocyst removal does not really illuminate the issue of target control over differentiation of presynaptic elements.

The present results suggest some specific and testable hypotheses about the role of cell interactions in shaping axonal endings. First, certain types of neurons (c.g., cochlcar ganglion cells) may have increased ability to produce different axonal ending types via interactions with different target neurons; this might be tested by, for example, inducing CN axons to form synapses with abnormal targets and comparing the resulting endings with those normally formed on that target cell. Second, it is possible that some neurons (e.g., NM neurons) form a single type of ending regardless of the target cell involved; this might be tested in the auditory system, for example, by transplating NM to ectopic sites or transplanting abnormal target neurons into the path of the contralaterally traveling branch of NM's axon and studying any contacts formed.

\section{Compensatory adaptations in synaptic form}

If one looks only at the axon terminal of the NM-NM sprout, it does not appear that interaction with an abnormal target has had much effect on its shape. Clearly, however, there has been a significant alteration in the relationship between pre- and postsynaptic elements in this anomalous, but functional and persistent, synapse.

The anomalous NM-NM projection arises from a population of neurons about half the size of the normal cochlear ganglion (Rubel and Parks, 1988) and the typical arbor of an anomalous NM axonal branch contains fewer than 10 boutonal endings (see Jackson and Parks, 1988) which are each only a fraction the size of a CN endbulb. Thus, the "coverage" of the target by the novel afferents falls considerably short of the coverage provided by the normal $\mathrm{CN}$ afferents; the present data suggest that operated neurons have only half the coverage of normal neurons 
at this age. Some adaptations of synaptic form in this novel functional contact may act to compensate partially for the reduction in afferent input.

In their interaction with NM neurons, the anomalous NM boutons have more than twice as much membrane in apposition to the target cell as in normal NM-NL contacts. That this is done by altering the physical relationship between the 2 elements rather than simply increasing the presynaptic element's size is unusual. In other cases of reactive synaptogenesis, the contact area between afferent and target cells is increased through formation of new axon terminal endings (e.g., Raisman, 1985) or through enlargement of the presynaptic membrane specializations on surviving afferent endings (Hillman and Chen, 1985). In their invagination by the target neuron, the anomalous NM endings most closely resemble the unusual normal axosomatic endings seen in the terminal nerve ganglia of teleost fishes (Matsutani et al., 1986). Such an intimate relationship could facilitate any kind of trophic interaction whose strength is proportional to the area of cell-cell contact. There is preliminary evidence (Parks et al., 1986) that one such interaction may be the induction in NM neurons of abnormal postsynaptic densities.

\section{References}

Adams, J. C. (1981) Heavy metal intensification of DAB-based HRP reaction product. J. Histochem. Cytochem. 29: 775.

Campbell, G., and D. O. Frost (1988) Synaptic organization of anomalous retinal projections to the somatosensory and auditory thalamus: Target-controlled morphogenesis of axon terminals and synaptic glomeruli. J. Comp. Neurol. 272: 383-408.

Code, R. A., and E. W. Rubel (1989) Glycine-immunoreactivity in the auditory brain stem of the chick. Hear. Res. 40:167-172.

Code, R. A., G. D. Burd, and E. W. Rubel (1989) Development of GABA-immunoreactivity in brainstem auditory nuclei of the chick: Ontogeny of gradients in terminal staining. J. Comp. Neurol. (in press).

Cotman, C. W. (1985) Synaptic Plasticity, New York, Guilford Press.

Escher, G., N. Schonenberger, and H. van der Loos (1983) Detergentsoaked HRP-chips: A new method for precise and effective delivery of small quantities of the tracer to nervous tissue. J. Neurosci. Methods 9: 87-94.

Goldberg, J. M., and W. E. Brownell (1973) Discharge characteristics of neurons in anteroventral and dorsal cochlear nuclei of cat. Brain Res. 64: 35-54.

Hackett, J. R., H. Jackson, and E. W. Rubel (1982) Synaptic excitation of the second- and third-order auditory neurons in the avian brain stem. Neuroscience $7: 1455-1469$.

Hillman, D. E., and S. Chen (1985) Plasticity in the size of presynaptic and postsynaptic membrane specializations. In Synaptic Plasticity, C. W. Cotman, ed., pp. 39-76, New York, Guilford.

Jackson H., and T. N. Parks (1982) Functional synapse elimination in the avian cochlear nucleus with simultaneous reduction in cochlearnerve axon branching. J. Neurosci. 2: 1736-1743.

Jackson, H., and T. N. Parks (1988) Induction of novel functional afferents to the chick cochlear nucleus. J. Comp. Neurol. 271: 106114.
Jhaveri, S. R., and D. K. Morest (1982a) Neuronal architecture in nucleus magnocellularis of the chicken auditory system with observations on nucleus laminaris: A light and electron microscopic study. Neuroscience 7: 809-835.

Jhaveri, S. R., and D. K. Morcst (1982b) Sequential alterations of neuronal architecture in nucleus magnocellularis of the developing chicken: A Golgi study. Neuroscience 7: 837-853.

Jhaveri, S. R., and D. K. Morest (1982c) Sequential alterations of neuronal architecture in nucleus magnocellularis of the developing chicken: An electron microscope study. Neuroscience 7: 855-870.

Lorente de No, R. (1981) The Primary Acoustic Nuclei, Raven, New York.

Matsutani, S., H. Uchiyama, and H. Ito (1986) Cytoarchitecture, synaptic organization and fiber connections of the nucleus olfactoretinalis in a teleost (Navodon modestus). Brain Res. 373: 126-138.

Mugnaini, E. (1971) Developmental aspects of synaptology with special emphasis on the cerebellar cortex. In Cellular Aspects of Neural Growth and Differentiation, D. C. Pease, ed., pp. 141-165, University of California Press, Los Angeles.

Nemeth, E. F., H. Jackson, and T. N. Parks (1985) Evidence for synaptic transmission mediated by kainate-preferring receptors in the avian cochlear nucleus. Neurosci. Lett. 59: 297-301.

Palay, S. L., and V. Chan-Palay (1974) The Cerebellar Cortex, Springer, New York.

Parks, T. N. (1979) Afferent influences on development of the avian brain stem auditory system: Otocyst ablation. J. Comp. Neurol. 183: 665-678.

Parks, T. N. (1981a) Changes in the length and organization of nucleus laminaris dendrites after unilateral otocyst ablation in chick embryos. J. Comp. Neurol. 203: 425-440.

Parks, T. N. (1981b) Morphology and origin of axosomatic endings in an avian cochlear nucleus: Nucleus magnocellularis of the chicken. J. Comp. Neurol. 203: 459-466.

Parks, T. N., and H. Jackson (1984) A developmental gradient of dendritic loss in the avian cochlear nucleus occurring independently of primary afferents. J. Comp. Neurol. 227: 459-466.

Parks, T. N., P. Collins, and J. W. Conlee (1983) Morphology and origin of axonal endings in nucleus laminaris of the chicken. J. Comp. Neurol. 214: 32-42.

Parks, T. N., D. Taylor, and H. Jackson (1986) Adaptations of synaptic form in a functional "sprouted" projection to the avian cochlear nucleus. Anat. Rec. 214:97A.

Parks, T. N., H. Jackson, and J. W. Conlee (1987) Axon-target cell interactions in the developing auditory system. Curr. Topics Dev. Biol. 21: 309-340.

Raisman, G. (1985) Synapse formation of adult rats. In Synaptic Plasticity, C. W. Cotman, ed., pp. 13-38, Guilford, New York.

Rubel, E. W., and T. N. Parks (1988) Organization and development of the avian brain stem auditory system. In Auditory Function: Neurobiological Bases of Hearing. G. M. Edelman et al., eds., pp. 3-92, Wiley, New York.

Ryugo, D. K., and D. M. Fekete (1982) Morphology of primary axosomatic endings in the anteroventral cochlear nucleus of the cat: A study of the endbulbs of Held. J. Comp. Neurol. 210: 239-257.

Schneider, G. E. (1981) Early lesions and abnormal neuronal connections. Trends Neurosci. 4: 187-192.

Young, S. R., and E. W. Rubel (1983) Frequency-specific projections of individual neurons in chick brainstem auditory nuclei. J. Neurosci. 3: 1373-1378.

Young, S. R., and E. W. Rubel (1986) Embryogenesis of arborization pattern and topography of individual axons in $\mathrm{n}$. laminaris of the chicken brain stem. J. Comp. Neurol. 254: 425-459. 